\title{
Nuclear Localization of Foamy Virus Gag Precursor Protein
}

\author{
ANDREAS W. SCHLIEPHAKE AND AXEL RETHWILM* \\ Institut für Virologie und Immunbiologie, Würzburg, Germany
}

Received 1 January 1994/Accepted 27 April 1994

\begin{abstract}
All foamy viruses give rise to a strong nuclear staining when infected cells are reacted with sera from infected hosts. This nuclear fluorescence distinguishes foamy viruses from all other retroviruses. The experiments reported here indicate that the foamy virus Gag precursor protein is transiently located in the nuclei of infected cells and this is the likely reason for the typical foamy virus nuclear fluorescence. By using the vaccinia virus expression system, a conserved basic sequence motif in the nucleocapsid domain of foamy virus Gag proteins was identified to be responsible for the nuclear transport of the gag precursor molecule. This motif was also found to be able to direct a heterologous protein, the Gag protein of human immunodeficiency virus, into the nucleus.
\end{abstract}

The foamy viruses are a poorly characterized subgroup of the family of retroviruses (42). Foamy viruses are ubiquitously present in simians, felines, and bovines but have only occasionally been found in other mammalian species $(17,18,28)$. Their natural prevalence among humans is a matter of controversy, but accidental infections by simian viruses indicate that humans are susceptible to foamy virus infections (38). The pathogenic potential of foamy viruses remains unresolved. Whereas mice transgenic for foamy virus genes developed a severe encephalopathy and myopathy (4), natural foamy virus infections are believed to result in lifelong persistence and infections of an apparently benign nature $(17,18,28)$.

In 1971 the isolation of a foamy virus from human tissue (HFV) was reported (1). This foamy virus is the only isolate of human origin that is available to the scientific community. HFV has been molecularly cloned and sequenced, and infectious molecular clones have been constructed $(12,27,31,43$, $44)$. In addition to gag, pol, and env, foamy viruses possess accessory open reading frames (ORF), termed bel, in the $3^{\prime}$ region of the genome which give rise to two accessory proteins, Bel-1 and Bet $(3,12,27,42)$. Other bel gene-derived proteins initially postulated on the basis of PCR mapping of splice sites (33) have yet to be unambiguously identified $(3,42)$.

In tissue culture, foamy viruses give rise to extensively vacuolated, multinucleated syncytia in a wide range of cells. These cells show a strong nuclear staining when they are reacted with the sera from foamy virus-infected hosts in an immunofluorescence assay (IFA) $(11,17-19,28,29,39)$. This nuclear fluorescence is a long-recognized hallmark of foamy viruses that has been observed with all virus isolates and all cell types that have been studied $(17,18,28,50)$. Since no such reaction is observed with any other retrovirus (50), the typical nuclear fluorescence of foamy viruses has been regarded as the best criterion for the serodiagnosis of foamy virus infection (38).

While the accessory Bet protein is localized to the cytoplasm, the HFV Bel-1 transactivator protein is a nuclear protein $(3,21)$. However, regulatory proteins of retroviruses are in general only required and synthesized in minute amounts and give rise to only weak, if any, immune response in the infected host $(20,40)$. By analogy, the cytoplasmic fluores-

\footnotetext{
* Corresponding author. Mailing address: Institut für Virologie und Immunbiologie, Versbacher Str. 7, 97078 Würzburg, Germany. Phone: (49) 931-201-5954. Fax: (49) 931-201-3934
}

cence that is observed when human immunodeficiency virus (HIV)-infected cells are reacted with sera from infected individuals, despite two nuclear-localized HIV regulatory proteins $(7,20,40)$, makes Bel-1 an unlikely candidate for the foamy virus nuclear fluorescence.

We therefore hypothesized that a structural foamy virus protein might be localized to the nuclei of infected cells and attempted to analyze the molecular basis of the foamy virus nuclear fluorescence.

\section{MATERIALS AND METHODS}

Cells and viruses. Diploid human embryonic lung fibroblasts (HEL and MRC-5), human glioma cells (U-87), human epitheloid cells (HeLa), human osteosarcoma cells (143B), mouse fibroblasts (3T3TK ${ }^{-}$), baby hamster kidney cells (BHK-21), and African green monkey kidney cells (Vero) were grown in minimal essential medium containing 5 to $10 \%$ fetal calf serum and antibiotics. HFV (1) and the related chimpanzee foamy virus (simian foamy virus type 6 [SFV-6]) $(16,19)$ were propagated on HEL cells and titered by infectious center assay as described previously (3). SFV-6 was kindly provided by $D$. Neumann-Haefelin (Freiburg, Germany). Vaccinia virus (VV), Copenhagen strain, was a gift of U. Brinckmann (Würzburg, Germany) and was grown and titered on Vero cells as previously described (30).

Recombinant DNA. All plasmid constructions were performed by standard techniques (47). All molecular clones were characterized by restriction enzyme analysis and/or DNA sequencing of the relevant parts by the dideoxy chain termination method (48).

The source of all HFV gag fragments inserted into the VV transfer vectors was pBgag-1. pBgag-1 covers the complete HFV gag gene as a 3.54-kb MstII fragment derived from the infectious HFV clone pHSRV (43) that was inserted into the EcoRV site of pBluescript; 2.84-kb NarI-AfIII, 1.97-kb NarIDraIII, 1.88-kb NarI-PvuII, 1.63-kb HhaI-HhaI, and 1.50-kb NarI-StuI fragments from pBgag-1 were individually inserted into the multiple cloning site of the VV transfer vector p7.5K131 (kindly provided by A. von Brunn, Munich, Germany) downstream of the $7.5 \mathrm{~K}$ promoter after the ends were flushed with Klenow enzyme or T4 polymerase where appropriate. These plasmids (pVgag-1 to pVgag-5) are depicted in Fig. 1.

The VV transfer plasmid pDK-1 (kindly provided by $D$. 


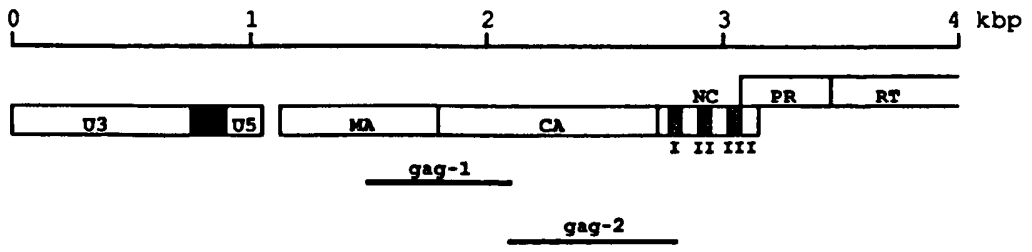

gag-3

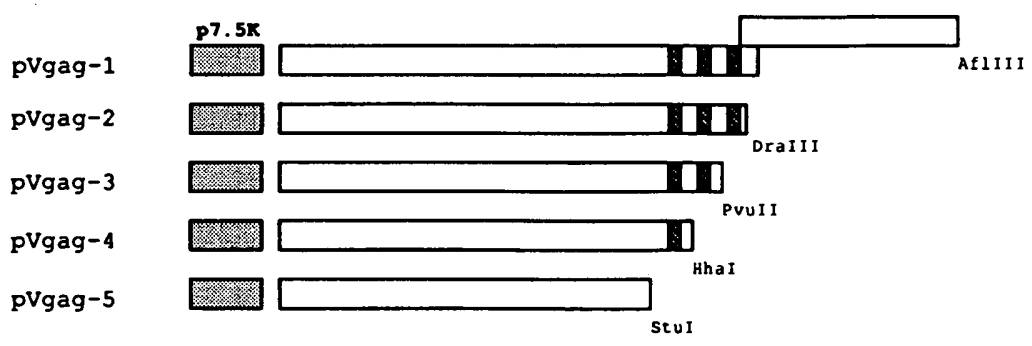

FIG. 1. HFV gag deletion mutants inserted into the VV transfer vector. The $5^{\prime}$ HFV genome organization and the gag fragments used for the generation of rabbit antisera (gag-1 to gag-3) are shown in the upper part of the figure. The glycine-arginine-rich (GR) boxes (I to III) in the proposed nucleocapsid (NC) domain of Gag are indicated. Matrix protein (MA), major capsid protein (CA), protease (PR), and reverse transcriptase (RT) are shown. $3^{\prime}$ deleted fragments of the HFV genome spanning gag and pol sequences as indicated in the lower part of the figure were inserted into $\mathrm{p} 7.5 \mathrm{~K} 131$ downstream of the VV $7.5 \mathrm{~K}$ promoter.

Kuritzkes, Denver, Colo.) encodes for the HIV-1 Gag protein (23). pDK-1 was cut at a single BgIII site after 437 of 500 codons of the HIV gag gene. Insertion of a double-stranded oligonucleotide providing a single $S t u I$ site and termination codons in all reading frames led to pAWS-1 (Fig. 2). pAWS-2 to pAWS-9 were generated by blunt end insertion of the 448-bp AccI-DraIII (pAWS-2), 361-bp AccI-PvuII (pAWS-3), 304-bp ScaI-DraIII (pAWS-4), 144-bp AccI-ScaI (pAWS-6), 161-bp ScaI-StyI (pAWS-7), 142-bp HaeIII-DraIII (pAWS-8), and 390-bp SspI-SspI (pAWS-9) fragments, respectively, into the StuI site of pAWS-1 in frame with the HIV gag coding sequence. To generate pAWS-5, the HFV gag 192-bp StuIDraI and 123-bp PvuII-PvuII fragments were first subcloned into the SmaI and EcoRI sites, respectively, of pBluescript, giving rise to $\mathrm{pBgag}-3$. Finally, a BamHI-EcoRV fragment from pBgag-3 was inserted into StuI-cut pAWS-1. Nucleotide sequencing of pAWS-2 to pAWS-9 revealed a continuous reading frame covering the HIV gag gene and the HFV gag fragments. The pAWS-1 to pAWS-9 plasmids are shown in Fig. 2.

pBgag-4 was derived by insertion of a $257-$ bp NcoI-StuI fragment of pBgag-1 into the PstI site of pBluescript via blunt-end ligation. The HFV gag 144-bp AccI-ScaI, 161-bp ScaI-StyI, and 142-bp HaeIII-DraIII fragments were individually cloned into the EcoRV site of pBgag-4 in frame with the already inserted HFV gag coding sequence, giving rise to pBgag-5 to -7 , respectively. Finally, the HFV gag motifs from pBgag-4 to -7 were excised as BamHI-HindIII fragments and inserted into the StuI site of pAWS-1 in frame with the HIV gag coding sequence, generating pAWS-10 and pAWS-12 to pAWS-14, respectively, as shown in Fig. 3. pAWS-11 was generated by inserting a 423-bp NcoI-ScaI fragment from pBgag-1 into pAWS-1.

Transfections, indirect immunofluorescence, and antibodies. Cells were grown on glass slides and infected when near to confluency with VV at a multiplicity of infection (MOI) of approximately 5 PFU per cell. Virus was allowed to adsorb for $1 \mathrm{~h}$ at $37^{\circ} \mathrm{C}$. After removal of the inoculum and one wash with complete minimal essential medium, the cells were transfected with the plasmids by using the DOTAP method as described by the manufacturer (Boehringer, Mannheim, Germany). In brief, 1 $\mu \mathrm{g}$ of plasmid DNA was diluted in $30 \mu \mathrm{l}$ of HBS $(20 \mathrm{mM} \mathrm{N}$. 2-hydroxyethylpiperazine- $N^{\prime}$-2-ethanesulfonic acid [HEPES], $15 \mathrm{mM} \mathrm{NaCl}$, [pH 7.4]) in a polystyrene reaction vial. A total of $10 \mu$ l of the transfection reagent DOTAP $(1 \mathrm{mg} / \mathrm{ml})$ was diluted separately in $30 \mu \mathrm{l}$ with HBS. Both solutions were mixed and incubated for $10 \mathrm{~min}$ at room temperature. After addition of $140 \mu \mathrm{l}$ of minimal essential medium, approximately $2 \times 10^{4}$ Vero cells were transfected with $100 \mathrm{ng}$ of plasmid DNA in a total volume of $20 \mu \mathrm{l}$ of transfection mixture and incubated for 3 to $5 \mathrm{~h}$ at $37^{\circ} \mathrm{C}$. After the transfection mixture was replaced by fresh medium, the cells were further incubated for 18 to $20 \mathrm{~h}$. For fixation the cells were washed in phosphatebuffered saline (PBS) and subsequently treated with $3.5 \%$ (vol/vol) formaldehyde in PBS for $30 \mathrm{~min}$ and $0.25 \%$ (vol/vol) Triton X-100 in PBS for 10 min, both at room temperature.

BHK-21 and HEL cells were grown on coverslips and infected with HFV or SFV-6 at an MOI of 10 . After $2 \mathrm{~h}$ for virus adsorption, the inoculum was replaced by fresh medium which, in some experiments, contained $0.2 \mu \mathrm{g}$ of actinomycin D (Sigma, Munich, Germany) per ml. After time intervals ranging from 0 to $96 \mathrm{~h}$ postinfection (p.i.), the cells were fixed in cold methanol.

IFA was carried out by blocking the cells with PBS-3\% fetal calf serum for 60 to $90 \mathrm{~min}$ at $37^{\circ} \mathrm{C}$. After incubation with rabbit- or mouse-derived first antibody for 60 to $90 \mathrm{~min}$ at $37^{\circ} \mathrm{C}$, the cells were washed twice in PBS $-0.25 \%$ Tween 20 . The appropriate fluorescein-conjugated second antibody (Dako, Hamburg, Germany) was added for 60 to $90 \mathrm{~min}$ at $37^{\circ} \mathrm{C}$. After extensive washing in PBS-Tween, the slides were mounted and examined under a Leitz Aristoplan microscope. IFA of VV-infected and transfected cells revealed an identical staining pattern of all cells transfected with a given plasmid.

The rabbit antisera gag-1, gag-2, and gag-3, directed against the matrix (MA), major capsid (CA), and nucleocapsid (NC) domains of the HFV Gag protein (Fig. 1), respectively, and $\mathrm{rt}-1, \mathrm{tm}-1$, bel-1, and bel-2, directed against the reverse tran- 


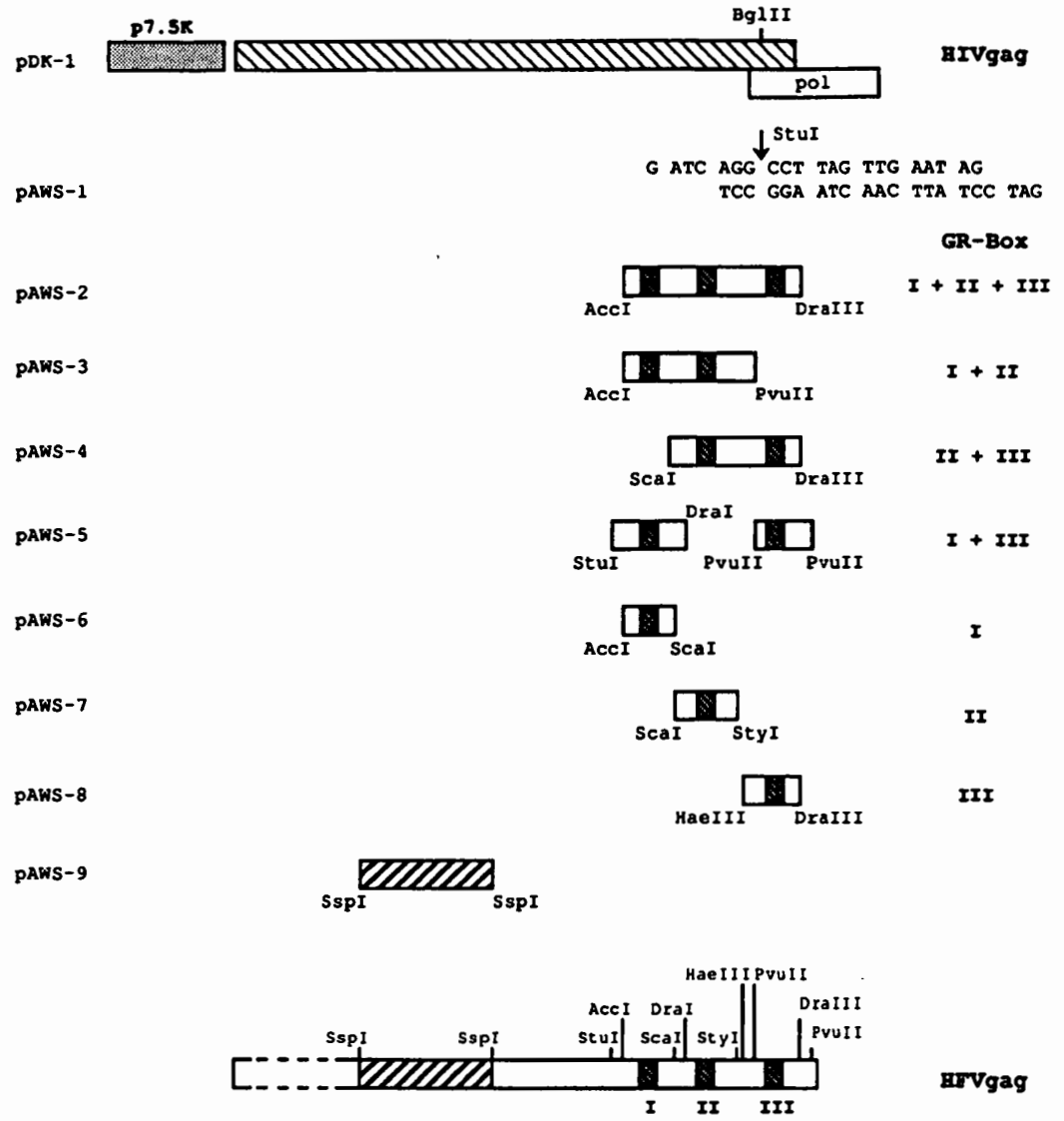

FIG. 2. Construction of a VV transfer vector expressing the HIV-1 Gag protein and insertion of HFV gag sequences. An oligonucleotide providing a single $S t u I$ cloning site was inserted into the unique BglII restriction site of the HIV-1 Gag protein encoding VV transfer plasmid pDK-1. HFV NC fragments covering the GR boxes in all possible combinations (pAWS-2 to pAWS-8) or a control fragment derived from the CA domain (pAWS-9) was inserted into the new transfer plasmid such that the HFV gag sequences came in frame with the HIV gag sequences.

scriptase, the transmembrane, the Bel-1, and the Bet proteins, respectively, have been described recently $(2,3,37)$. For immunofluorescence, gag and bel antisera were diluted 1:200 in PBS $-0.1 \%$ bovine serum albumin, while the pol and env antisera were diluted 1:50. To detect HIV-1 Gag protein, a mouse monoclonal antibody directed against p24 CA (Dianova, Hamburg, Germany) was used at a 1:40 dilution. The human serum which contains antibodies against the HFV Gag, Pol, Env, Bel-1, and Bet proteins has already been described (36) and was used at a 1:100 dilution.

Cell fractionation and immunoblot. MRC-5 cells infected with HFV at a MOI of 10 for $48 \mathrm{~h}$ were separated into cytoplasmic and nuclear fractions by a procedure described previously (24) with slight modifications. Briefly; the cells were washed three times with PBS, overlaid with hypotonic buffer (10 mM HEPES, $10 \mathrm{mM} \mathrm{NaCl}, 1 \mathrm{mM} \mathrm{MgCl}$, $1 \mathrm{mM}$ phenylmethylsulfonyl fluoride, $1 \mu \mathrm{M}$ pepstatin $\mathrm{A}, 0.1 \mu \mathrm{M}$ aprotinin, $10 \mu \mathrm{M}$ protease inhibitor E64 [Sigma], $\mathrm{pH}$ 6.2) for $15 \mathrm{~min}$. After a scraping, the cells were passed 10 times through a 20-gauge needle. Cytoplasmic and nuclear fractions were separated by centrifugation at $1,000 \times g$ for $15 \mathrm{~min}$ and at $4^{\circ} \mathrm{C}$ The pellet (nuclei) was resuspended in a buffer containing 50 $\mathrm{mM}$ Tris- $\mathrm{HCl}$ ( $\mathrm{pH} \mathrm{9.0),} 150 \mathrm{mM} \mathrm{NaCl}, 1 \%$ Nonidet $\mathrm{P}-40$, and protease inhibitors as described for the hypotonic buffer. The protein concentrations were determined with a commercial assay (Sigma), and equal amounts of proteins from each fraction were separated in a sodium dodecyl sulfate (SDS)containing $10 \%$ polyacrylamide gel. The gel was semidry blotted onto nitrocellulose membrane (Schleicher \& Schüll, Dassel, Germany), and immunoreaction was carried out with the gag-2 antibody as previously described, with the ECL chemiluminescence detection system (Amersham, Braunschweig, Germany) (3). Control lanes were reacted with a mouse monoclonal antibody directed against glyceraldehyde3-phosphate dehydrogenase (GAPDH), which was a gift of H. J. Degen, Würzburg, Germany.

\section{RESULTS}

The foamy virus Gag precursor protein is localized to the nuclei of infected cells. The reaction of foamy virus infected cells with serum from an infected individual gives rise to a bright nuclear staining (Fig. 4A), indicating a nuclear-localized immunodominant virus protein. To investigate whether a structural foamy virus protein is localized to the nuclei of infected cells, proliferating BHK-21 cells were infected at a high MOI to analyze the virus protein expression pattern in a single round of replication. Under these conditions the cells remained viable until signs of degradation became visible by light microscopy between 96 and $112 \mathrm{~h}$ after infection. Cells 


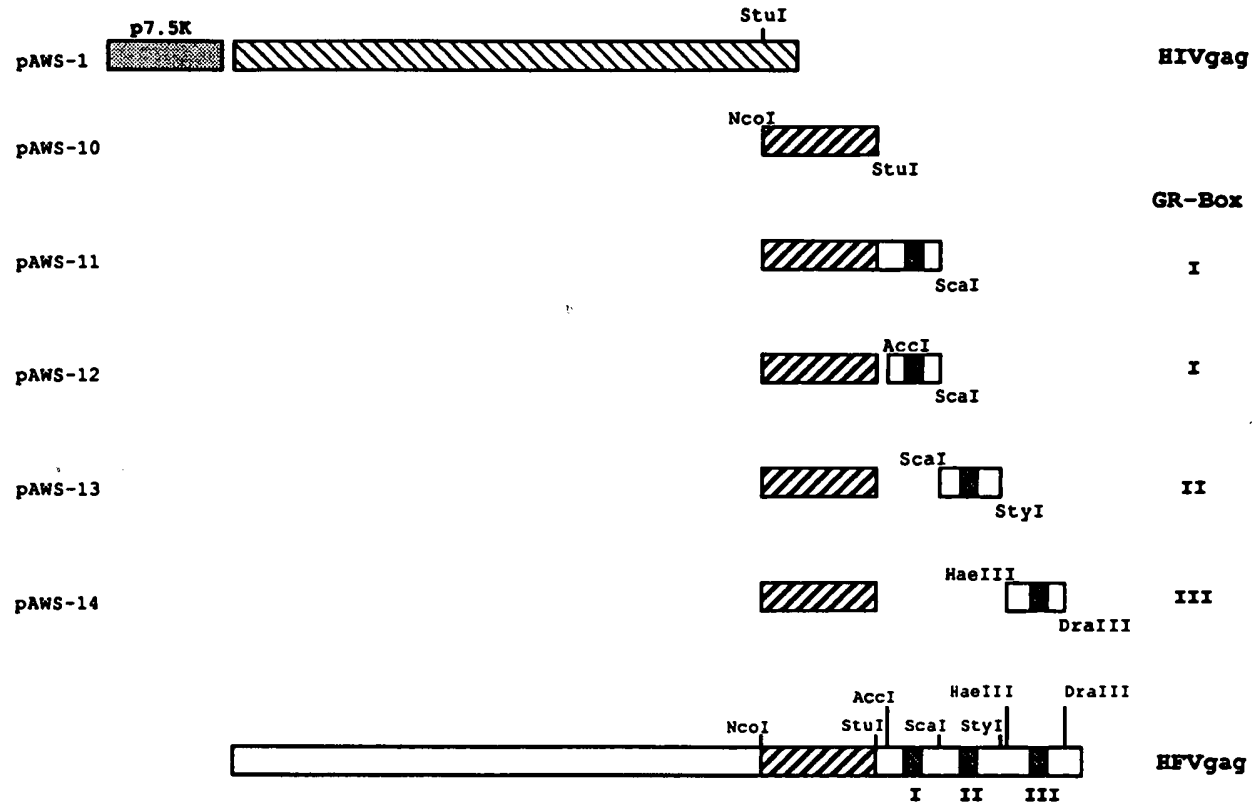

FIG. 3. Construction of VV transfer plasmids expressing HIV-HFV hybrid gag sequences to resolve the role of GR box I for the nuclear localization of HFV Gag protein. The HFV GR boxes I to III were individually inserted into pAWS-1 together with a fragment from the HFV gag sequence that precedes the basic motifs.

were infected at an MOI of 10 , fixed after time intervals ranging from 0 to $96 \mathrm{~h}$ p.i., and stained with rabbit antisera specific for the HFV Gag, Pol, and Env proteins for IFA. Env protein was first observed $36 \mathrm{~h}$ p.i. and was only detected in the cytoplasm of infected cells throughout the observation period (Fig. 4H). Pol protein became first detectable at $18 \mathrm{~h}$ p.i. and was predominantly observed in the cytoplasm (Fig. 4G). However, in later stages of infection (between 48 and $72 \mathrm{~h}$ p.i.), staining with Pol-specific antibody in addition revealed some weak nuclear staining in approximately $10 \%$ of infected cells (data not shown). By contrast, the HFV Gag protein behaved differently from Pol and Env. Gag protein was first detected 15 h p.i., when it localized solely to the cytoplasm (Fig. 4C). At 18 h p.i. gag antiserum began to stain the nuclei. At $48 \mathrm{~h}$ p.i., all Gag protein was observed in the nuclei of infected cells (Fig. 4D). At $72 \mathrm{~h}$ p.i., the nuclear fluorescence began to weaken and gag-specific antisera again stained the cytoplasm in approximately $50 \%$ of the infected cells (Fig. 4E). The transient nuclear localization of HFV Gag protein was observed with rabbit antisera generated against different domains of the HFV Gag protein (Fig. 1), indicating that newly synthesized Gag precursor protein is transported to the nuclei of infected cells. This view is supported by an experiment carried out to exclude the possibility that $\mathrm{Gag}$ protein from virions entering the cells gives rise to the nuclear fluorescence. When BHK-21 cells were infected at an $\mathrm{MOl}$ of 50 in the presence of actinomycin D (0.2 $\mu \mathrm{g} / \mathrm{ml}$ ), no staining with gag antibody was observed at any time point p.i. (Fig. 4F). Infection of BHK-21 cells with SFV-6 and staining with the cross-reactive antiserum directed against the HFV CA protein (gag-2) revealed the nuclear presence of SFV-6 Gag protein (Fig. 4B), demonstrating that different foamy virus isolates behave similarly with respect to the Gag nuclear localization. When primary human fibroblasts (HEL) were used instead of BHK-21 cells in infection experiments, HFV Gag protein was again observed in the nuclei of infected cells, demonstrating that the nuclear pathway of foamy virus
Gag protein is not a peculiarity of BHK-21 cells (data not shown).

Staining of infected cells with bel-1- and bel-2-specific antisera revealed a fluorescence pattern already described previously (3). Bel-1 was the first virus protein to become detectable by IFA after $12 \mathrm{~h}$ p.i. and localized to the nuclei of infected cells. However, at this time, reaction with foamy virus-positive primate sera did not show any staining (data not shown), indicating that the immune response against the Bel-1 regulatory protein is insufficient to give rise to the nuclear fluorescence observed with sera from infected individuals as shown in Fig. 4A. Furthermore, with the appearance of the cytoplasmic Bet protein which is made up of sequences of the bel-1 and bel-2 ORFs $(3,33) 15$ to 24 h p.i., bel-1 and bel-2 antisera stained the cytoplasms of infected cells (data not shown).

To further demonstrate that uncleaved Gag precursor protein is present in the nuclei, we fractionated cytoplasm from nuclei of diploid human fibroblasts (MRC-5) infected for $48 \mathrm{~h}$ with HFV at an MOI of 10. Equal amounts of protein from both fractions were separated by SDS-polyacrylamide gel electrophoresis (PAGE) and subjected to immunoblot reaction with gag- 2 antiserum directed against $C A$ protein. As shown in Fig. 5, the Gag precursor molecules of 70 and $74 \mathrm{kDa}$ (2) were easily detected in the nuclear fraction, while they were almost undetectable in the cytoplasm, as was the cleaved $32-\mathrm{kDa} C A$ protein in both fractions $(2,3)$. Staining with an antibody against the cytosolic GAPDH protein was performed as a control to ensure that protein was present in the cytoplasmic fraction.

Identification of a NLS in HFV Gag by the subtractive approach. The foamy virus $G$ ag proteins differ from the respective proteins of all other retroviruses in that they do not have cysteine-histidine boxes ( $\mathrm{CH}$ boxes) in their NC domains $(16,22,31,41)$. Instead, the proposed foamy virus NC domains harbor three stretches of highly basic glycine-arginine-rich boxes (GR boxes) (see Fig. 9). Since positively charged amino 

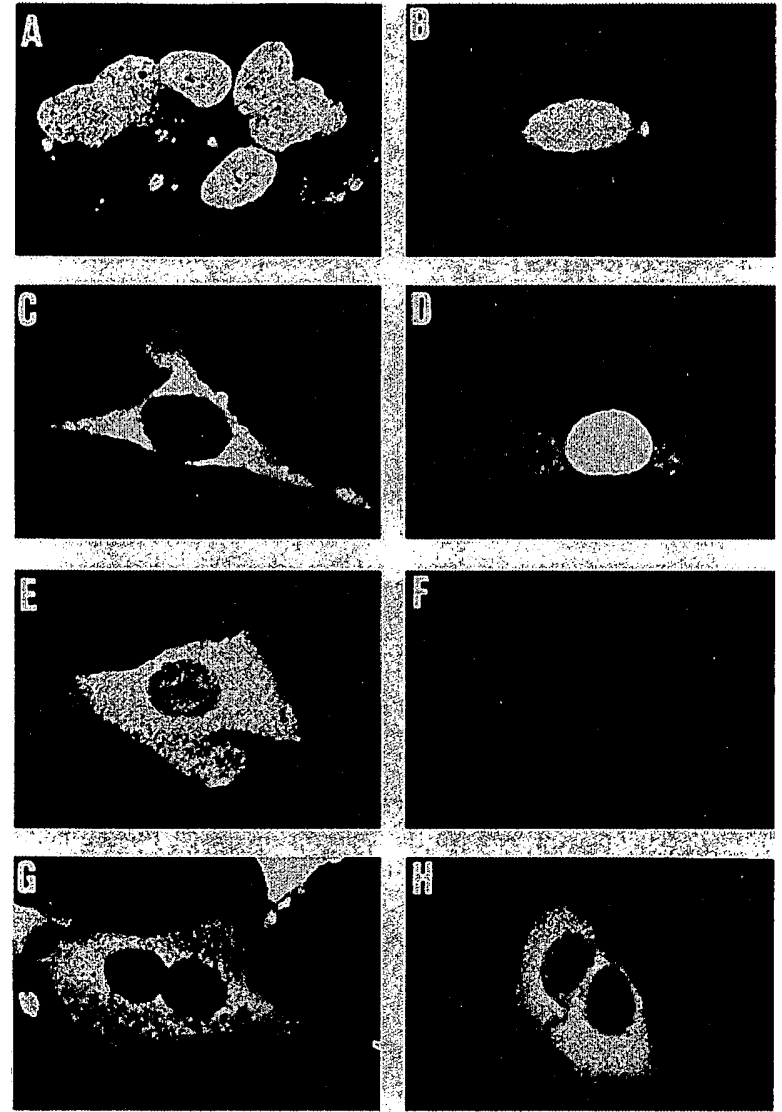

FIG. 4. IFA of the subcellular localization of foamy virus structural proteins in infected BHK-21 cells. (A, C, D, E, G, and H) Cells were infected with HFV at an MOI of 10 . Cells were fixed $48 \mathrm{~h}$ p.i. and reacted with HFV-positive human serum (A); cells were fixed 15 (C), 48 (D) and 72 (E) h p.i., respectively, and reacted with anti-HFV CA (gag-2) rabbit serum; or cells were fixed $48 \mathrm{~h}$ p.i. and reacted with anti-HFV pol $(G)$ and anti-HFV env $(\mathrm{H})$ rabbit sera. (B and F) Cells were reacted with gag-2 antiserum; (B) Cells were infected with the chimpanzee foamy virus SFV-6; (F) cells were infected with HFV at a $\mathrm{MOI}$ of 50 in the presence of actinomycin $D$ and fixed $2 \mathrm{~h}$ p.i.

acid sequences are often found in nuclear localization signals (NLS) (14), we investigated this possibility for the HFV Gag protein in order to analyze the molecular basis of the foamy virus Gag nuclear fluorescence. To do this, we inserted Cterminally truncated fragments of the HFV gag gene into a VV transfer vector (Fig. 1). When pVgag-1 or -2 harboring the three GR boxes was transfected into VV-infected Vero cells and subsequently stained with gag antibody in IFA, a strong nuclear fluorescence was observed (Fig. 6B and C), indicating that the VV-expressed $\mathrm{Gag}$ protein behaved in a manner similar to that of the foamy virus-expressed protein. The analysis of VV constructs in which the HFV gag gene was truncated after each of the GR boxes revealed that the region covering GR box II (pVgag-3 in Fig. 1; Fig. 6D) is required for the nuclear transport of foamy virus Gag protein, since $\mathrm{pV}$ gag-4 and -5 gave rise only to a cytoplasmic fluorescence (Fig. $6 \mathrm{E}$ and $\mathrm{F}$ ). When pVgag-3- and pVgag-4-transfected cells were analyzed with HFV-positive human serum, the same fluorescence pattern was observed as was seen in the reaction with gag antiserum (Fig. 6G and $\mathrm{H}$ ).

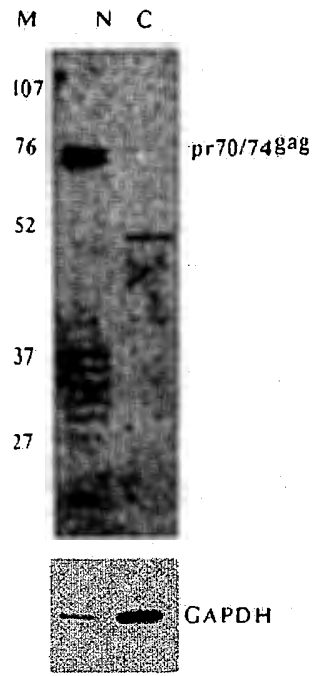

FIG. 5. Uncleaved Gag precursor protein is present in the nuclei of foamy virus-infected cells. MRC-5 diploid human fibroblasts were infected with HFV at an MOI of 10 for $48 \mathrm{~h}$. Cells were fractionated into cytoplasm (C) and nuclei (N), and proteins were separated by SDS-PAGE and reacted with antibody directed against HFV CA protein (gag-2) in the immunoblot assay. The uncleaved Gag precursors (pr70/74ag) are detectable in the nuclear fraction. Control lanes were reacted with an antibody against GAPDH. M, molecular mass markers (in kilodaltons).

Confirmation of the NLS by the additive approach. In the experiments described above the requirement of GR box II for the nuclear localization of HFV Gag protein was shown. To confirm that GR box II is also sufficient to serve as a NLS for a heterologous protein, we used the following additive approach. The VV transfer plasmid, pDK-1, encoding for the HIV-1 Gag protein, was modified by oligonucleotide linker insertion such that HFV gag fragments covering the GR boxes could be inserted in frame to the HIV gag coding sequence. The new plasmid pAWS-1 (Fig. 2) lacks the C-terminal 63 amino acids of the HIV Gag, but it retains the HIV CH boxes. Transient transfection of both plasmids, pDK-1 and pAWS-1, resulted in HIV-1 Gag protein expression in the cytoplasm (Fig. 7A and B).

As shown in Fig. 2, HFV gag fragments with the GR boxes in all possible combinations were introduced into pAWS-1. When the resulting plasmids, pAWS-2 to pAWS-9, were analyzed for the subcellular localization of the Gag fusion proteins, using a p24 monoclonal antibody, all constructs harboring the HFV GR box II gave rise to nuclear staining (Fig. 7C, D, E, and H). pAWS-8 harboring only GR box III, as well as pAWS-9 harboring a fragment derived from the HFV CA coding sequence (Fig. 2), resulted in the cytoplasmic expression of the respective fusion proteins (Fig. $7 I$ and $K$ ). Curiously, the recombinant Gag protein with GR box I expressed by pAWS- 6 was localized to the nuclei of transfected cells and, in particular, to the nucleoli (Fig. 7G), as revealed by double-staining experiments with anti-rRNA antibody (data not shown). In contrast, pAWS-5, which harbors GR box I in addition to box III (Fig. 2), led to a cytoplasmic appearance of the recombinant $\mathrm{Gag}$ protein (Fig. 7F).

While these experiments demonstrated that GR box II can serve as a NLS in a heterologous protein context, the role of box $I$ in nuclear localization of the $\mathrm{Gag}$ proteins remained 

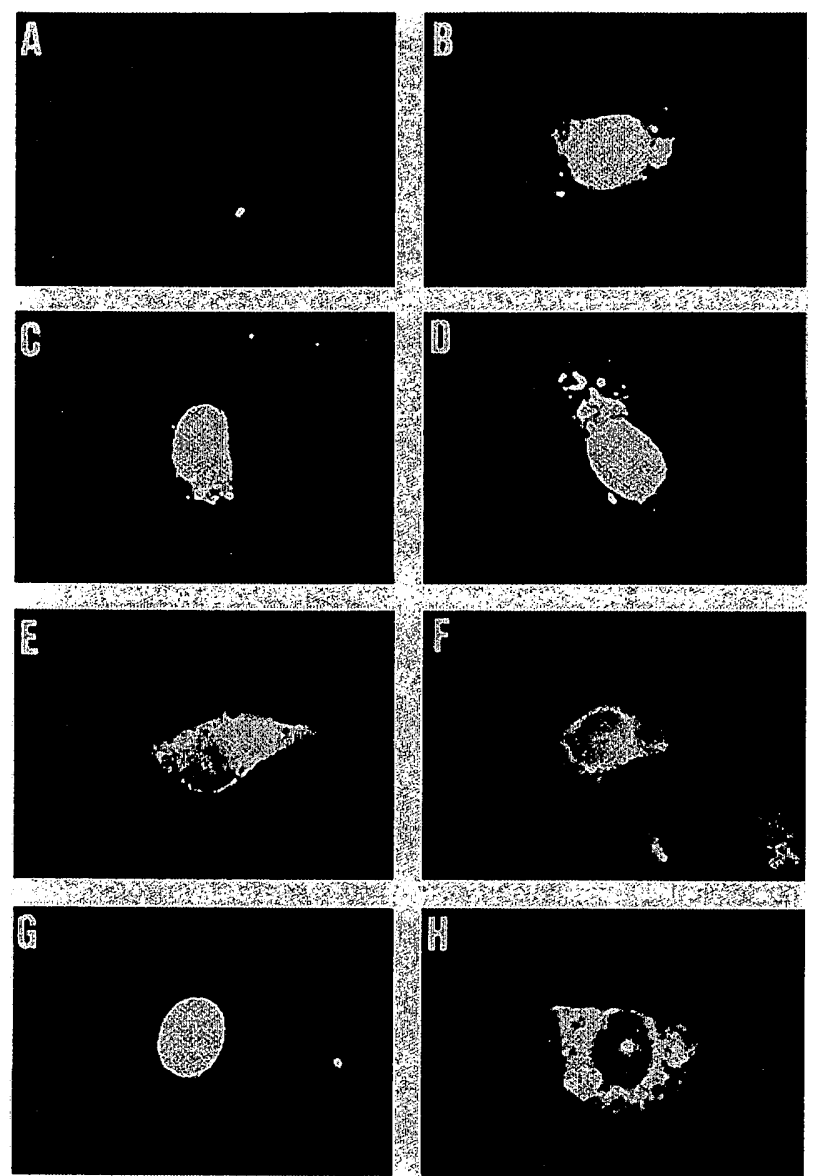

FIG. 6. IFA of the subcellular localization of HFV Gag deletion mutants expressed by VV transfer plasmids. The following plasmid DNAs were transfected into VV-infected Vero cells which were stained with gag-2 antibody: p7.5K131 (A), pVgag-1 (B), pVgag-2 (C), pVgag-3 (D), pVgag-4 (E), and pVgag-5 (F). pVgag-3-transfected cells (G) and pVgag-4-transfected cells (H) were reacted with human HFV-positive serum (36). Nuclear staining was observed only with constructs harboring $G R$ box II (B, C, D, and $G$ ).

obscure. GR box I, present in the constructs pVgag- 4 and pAWS-5, was found to be insufficient to locate HFV Gag protein as well as HIV Gag protein to the nucleus. This finding suggested that GR box I does not serve as a NLS in its natural context and that the nucleolar accumulation of the Gag protein observed with pAWS- 6 might be due to the strong RNAbinding properties of this particular recombinant, similar to previous observations of other systems which overexpress viral RNA-binding proteins (45).

To further analyze the contribution of GR box I to the nuclear localization of HFV Gag protein, we constructed an additional set of recombinants as depicted in Fig. 3. pAWS-10 harbors a HFV gag fragment preceding the GR boxes which was fused to the HIV gag coding sequence. In pAWS-11 to pAWS-14, the GR boxes were individually cloned downstream of this HIV/HFV hybrid gag gene. The analysis of the subcellular localization of the Gag proteins expressed from these constructs revealed that only pAWS-13, which harbors GR box II, gave rise to a nuclear fluorescence (Fig. 8C). This result indicates that GR box I does not act as a NLS in the context of the HFV Gag protein. The use of BHK-21 cells instead of
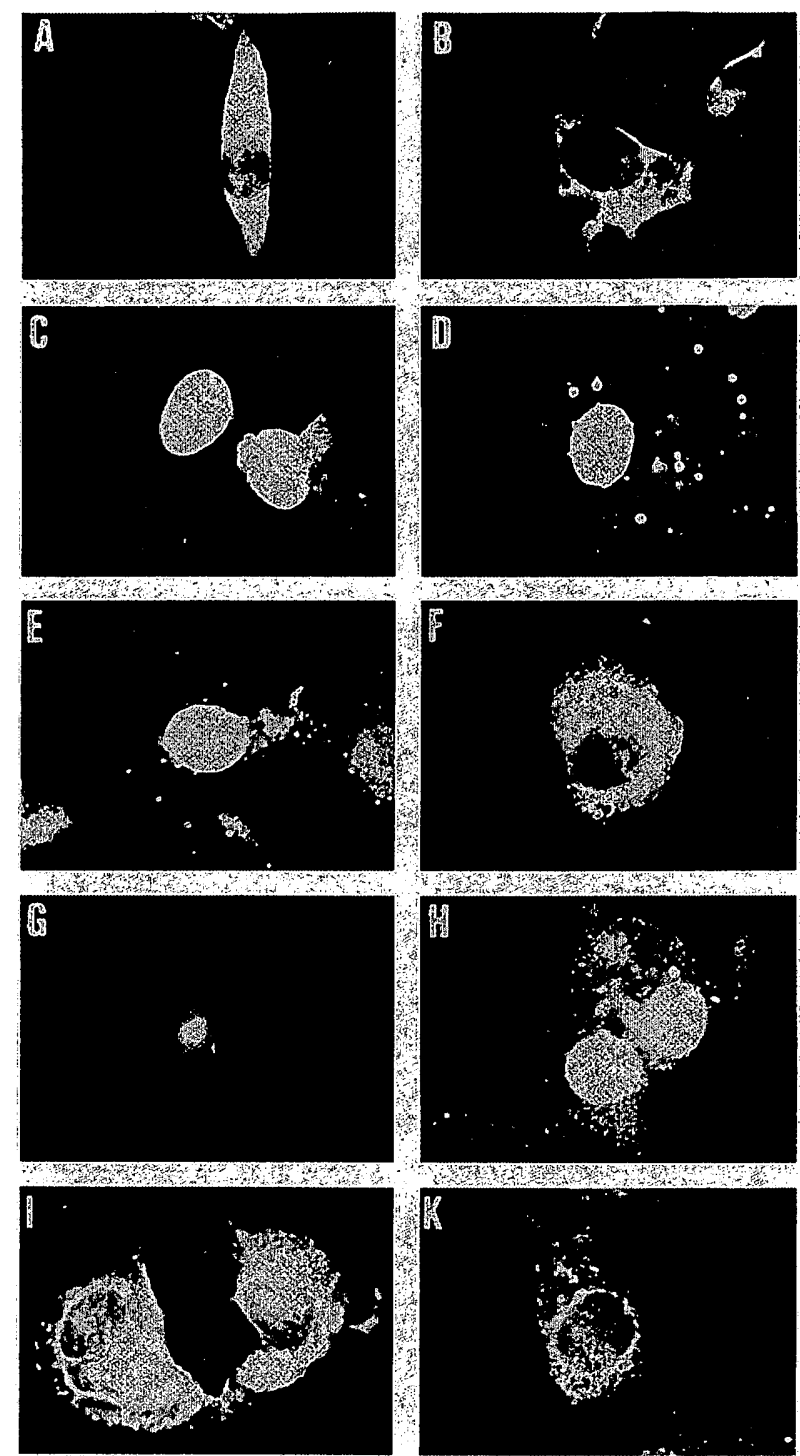

FIG. 7. IFA of the subcellular localization of hybrid HIV-HFV Gag proteins with anti-p24 monoclonal antibody. (A) pDK-1; (B) pAWS-1; (C) pAWS-2; (D) pAWS-3; (E) pAWS-4; (F) pAWS-5; (G) pAWS-6; (H) pAWS-7; (I) pAWS-8; (K) pAWS-9. While the authentic HIV Gag protein was localized to the cytoplasm (A and B), HIV-HFV Gag fusion proteins were located in the nucleus if the protein contained GR box II (C, D, E, and H). pAWS-5, containing GR boxes I and III, led to the cytoplasmic appearance of the fusion protein. Transfection of pAWS- 6 in which only GR box I was C terminally fused to the HIV gag coding sequence resulted in a predominantly nucleolar staining. Expression analysis of pAWS-7 transfected into 143B, HeLa, U87, or 3T3 cells gave identical results, as shown here for Vero cells, demonstrating the cell type independence of the foamy virus NLS (data not shown).

Vero cells resulted in an identical staining pattern with all $\mathrm{VV}$ constructs (data not shown).

\section{DISCUSSION}

The experiments reported here strongly suggest that the foamy virus typical nuclear fluorescence that is observed in 

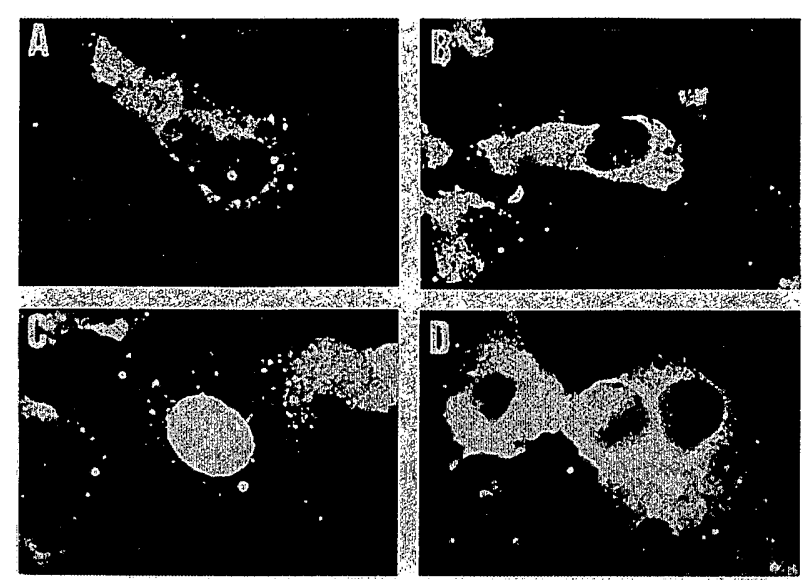

FIG. 8. IFA of the subcellular localization of hybrid HIV-HFV Gag proteins in Vero cells. (A) pAWS-10; (B) pAWS-12; (C) pAWS13; (D) pAWS-14. Only pAWS-13 (C) expressing a hybrid Gag protein with GR box II gave rise to a nuclear staining, while the other constructs led to the cytoplasmic accumulation of the fusion proteins. pAWS-11 gave results identical to those of pAWS-12 (data not shown). The cytoplasmic fluorescence observed with constructs pAWS-11 and pAWS-12 (B) indicated that GR box I does not serve as a NLS in its natural protein context.

IFA with sera from infected hosts is due to an antibody response against $\mathrm{Gag}$ antigen. The $\mathrm{Gag}$ protein precursor of foamy viruses was found to be transiently located in the nuclei of infected cells. By using the VV expression system, a basic region (GR box II) in the proposed NC domain of foamy virus Gag proteins was identified to be responsible for directing the Gag precursor protein into the nuclei of infected cells. Furthermore, GR box II was able to translocate a heterologous retroviral Gag precursor protein which is normally found only in the cytoplasm into the nucleus.

In foamy viruses the Gag proteins show a higher degree of genetic divergence than the other structural proteins (16, 22, $31,41)$. Alignment of the deduced NC sequences of four primate foamy virus isolates revealed a high degree of conservation of the three GR boxes, with GR box II being almost perfectly conserved, underlining the importance of this motif (Fig. 9). Furthermore, the primary structure of GR box II shows close similarities to NLS known from other proteins (for a review, see reference 14 ).

The lack of $\mathrm{CH}$ boxes, the conservation of the GR box II, and the observation that nuclear localization of Gag is a common feature of all foamy viruses investigated so far are suggestive of a specific function of the nuclear pathway of foamy virus Gag precursors in the virus replication cycle. However, at the moment one can only speculate on these possible functions.

Nuclear localization of retroviral Gag proteins in HIV has been described $(5,6,49)$. In this case the MA protein is believed to direct the nucleoprotein complex, after the uncoating of the virus, to the nucleus to deliver the $\operatorname{cDNA}(5,6,49)$. This finding for HIV is clearly different from that reported here because the nuclear translocation of foamy virus Gag protein takes place after synthesis and affects the Gag precursor.

Also, the nuclear localization of the foamy virus Gag precursor does not seem to be simply a side effect of unspecific RNA-binding properties of the protein. To our knowledge, no other retrovirus transports its Gag precursor quantitatively to

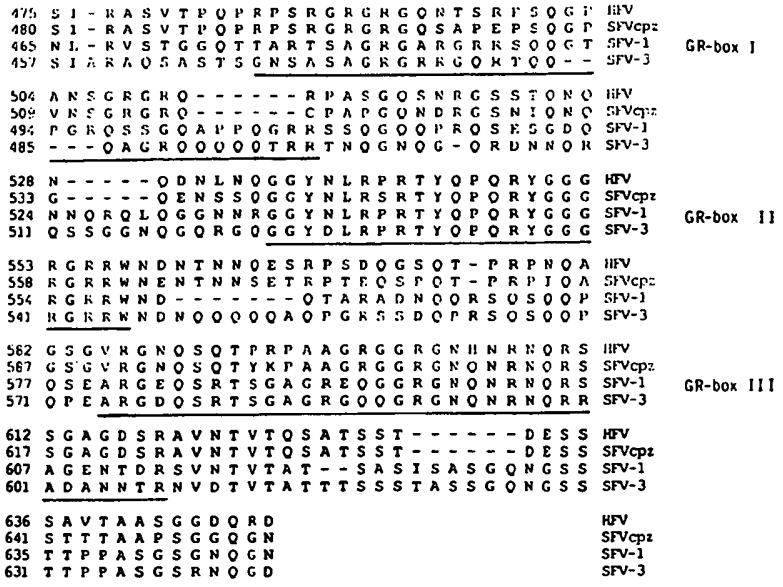

FIG. 9. Comparison of the C-terminal Gag sequences derived from four different primate foamy viruses demonstrates the conservation of GR box II. Foamy virus Gag sequences $(16,22,31,41)$ from HFV, SFV-1, SFV-3, and from a foamy virus isolated from a chimpanzee (SFVcpz) were aligned by using the Megaline program (DNASTAR). The GR boxes are indicated. GR box II harbors the NLS.

the nucleus, although all retroviral Gag proteins bind RNA $(50,53)$. The cytoplasmic localization of VV-expressed HIV Gag protein (Fig. 7A) and the nuclear localization of VVexpressed HFV Gag protein described in this study (Fig. 6B) illustrate this fundamental difference. Unspecific nuclear localization may be the case with construct pAWS-6, in which the GR box I was fused C terminally to the HIV-1 Gag protein. The results obtained with plasmids pVgag-4, pAWS-5, pAWS11 , and pAWS-12 indicate that GR box I does not serve as a NLS in its natural protein context. The effect of the protein context on the function of a NLS has also been described for the simian virus 40 large $T$ nuclear signal (46). However, GR box I may have an enhancing effect on the nuclear transport of the HFV Gag precursor protein similar to that of HBV core protein (10).

A variety of hypotheses may explain the nuclear pathway of the foamy virus Gag precursor, each having arguments for and against.

The primary structure of foamy virus NC domains is reminiscent of the class of arginine-rich RNA-binding proteins (25) and the C-terminal basic motif of hepatitis B virus (HBV) core protein $(10,34,35)$. With respect to $\mathrm{HBV}$, it is noteworthy that the core protein is localized, at least in part, to the nuclei of infected cells $(10,13,35)$. It is believed that HBV core particles perform an intracellular replication cycle. This replicative shortcut was suggested to result in the accumulation of HBV DNA in the infected cell $(13,35,52)$. Large amounts of unintegrated DNA have also been reported for foamy viruses (32). It remains to be seen whether or not this finding can be attributed to a similar mechanism, as hypothesized for HBV. In general, large amounts of unintegrated DNA in retrovirusinfected cells are believed to result from superinfection (51).

Packaging of retroviral genomic RNA occurs in the cytoplasm $(26,53)$. The lack of the otherwise conserved $\mathrm{CH}$ boxes in the NC domain and the presence of the GR boxes are indicative of a different mechanism of RNA packaging in foamy viruses compared with that in other retroviruses (26, 53). Assuming that the GR boxes take over the role of the $\mathrm{CH}$ boxes in packaging, this may involve the pickup of the genomic RNA in the nucleus. The transiency of the nuclear-localized 
foamy virus Gag precursor protein is a strong argument for this hypothesis. In this case one might expect foamy virus core particles to assemble in the nucleus. Nuclear-localized core structures have been occasionally observed in SFV-1- and BFV-infected cells by electron microscopy $(8,9)$. However, the nuclear presence of core particles is not a general characteristic of foamy viruses, and immature viral cores are easily observed in the cytoplasms of infected cells (15).

Finally, one may consider a regulatory function for foamy virus $\mathrm{Gag}$ proteins. In contrast to other complex retroviruses, a posttranscriptionally acting regulatory protein that directs the expression of structural proteins in foamy viruses has not been identified (42). We therefore cannot exclude that the foamy virus Gag protein acts as a positive regulatory of the expression of the genomic RNA. However, previous studies indicate that there is no evidence for such a mechanism in foamy viruses (3). Future experiments will have to clarify whether or not the nuclear pathway of the Gag precursor is essential for foamy virus replication and what its specific function is.

\section{ACKNOWLEDGMENTS}

We thank U. Brinckmann, A. von Brunn, H. J. Degen, D. Kuritzkes, and D. Neumann-Haefelin for the gift of reagents; $O$. Herchenröder for communicating the SFVcpz gag sequence prior to publication; B. Geyer for help with the VV system; S. Kaltenthaler for anti-rRNA serum; S. Siddell for help with the computer analysis; and L. Dunster for critical reading of the manuscript.

This work was supported by the Deutsche Forschungsgemeinschaft (SFB 165) and the EU (BMH1-CT93-1142).

\section{REFERENCES}

1. Achong, B. G., P. W. A. Mansell, M. A. Epstein, and P. Clifford. 1971. An unusual virus in cultures from a human nasopharyngeal carcinoma. J. Natl. Cancer. Inst. 46:299-307.

2. Aguzzi, A., E. F. Wagner, K.-O. Netzer, K. Bothe, I. Anhauser, and A. Rethwilm. 1993. Human foamy virus proteins accumulate in neurons and induce multinucleated giant cells in the brain of transgenic mice. Am. J. Pathol. 142:1061-1072.

3. Baunach, G., B. Maurer, H. Hahn, M. Kranz, and A. Rethwilm. 1993. Functional analysis of human foamy virus accessory reading frames. J. Virol. 67:5411-5418.

4. Bothe, K., A. Aguzzi, H. Lassmann, A. Rethwilm, and I. Horak. 1991. Progressive encephalopathy and myopathy in transgenic mice expressing human foamy virus genes. Science 253:555-557.

5. Bukrinsky, M. J., S. Haggerty, M. P. Dempsey, N. Sharova, A. Adzhubei, L. Spitz, P. Lewis, D. Goldfarb, M. Emerman, and M. Stevenson. 1993. A nuclear localization signal within the HIV-1 matrix protein that governs infection of non-dividing cells. Nature (London) 356:666-669.

6. Bukrinsky, M. J., N. Sharova, M. P. Dempsey, T. L. Stanwick, A. G. Bukrinskaya, S. Haggerty, and M. Stevenson. 1992. Active nuclear import of human immunodeficiency virus type 1 preintegration complexes. Proc. Natl. Acad. Sci. USA 89:6580-6584.

7. Cullen, B. R. 1991. Regulation of human immunodeficiency virus replication. Annu. Rev. Microbiol. 45:219-250.

8. Dermott, E., J. K. Clarke, and J. Samuels. 1971. The morphogenesis and classification of bovine syncytial virus. J. Gen. Virol. 12:105-119.

9. Dermott, E., and J. Samuels. 1973. Intrachromosomal location of MK5, a foamy type 1 virus. J. Gen. Virol. 19:141-143.

10. Eckhardt, S. G., D. R. Milich, and A. McLachlan. 1991. Hepatitis $B$ virus core antigen has two nuclear localization sequences in the àrginine-rich carboxyl terminus. J. Virol. 65:575-582.

11. Fleming, W. A., and J. K. Clarke. 1970. Fluorescence assay of foamy virus. J. Gen. Virol. 6:277-284.

12. Flügel, R. M., A. Rethwilm, B. Maurer, and G. Darai. 1987. Nucleotide sequence analysis of the env gene and its flanking regions of the human spumaretrovirus reveals two novel genes. EMBO J. 6:2077-2084.

13. Ganem, D. 1991. Assembly of hepadnaviral virions and subviral particles. Curr. Top. Microbiol. Immunol. 168:61-83.

14. Garcia-Bustos, J., J. Heitman, and M. N. Hall. 1991. Nuclear protein localization. Biochim. Biophys. Acta 1071:83-101.

15. Gelderblom, H., and H. Frank. 1987. Spumavirinae, p. 305-311. In M. V. Nermut and A. C. Steven (ed.), Animal virus structure. Elsevier, Amsterdam.

16. Herchenröder, O., R. Renne, D. Loncar, K. E. Cobb, K. K. Murphy, J. Schneider, A. Mergia, and P. A. Luciw. 1994. Isolation, cloning, and sequencing of simian foamy viruses from chimpanzees (SFVcpz): high homology to human foamy virus (HFV). Virology 201:187-199.

17. Hooks, J. J., and B. Detrick-Hooks. 1981. Spumavirinae: foamy virus group infections: comparative aspects and diagnosis, $p$ 599-618. In E. Kurstak and C. Kurstak (ed.), Comparative diagnosis of viral diseases, vol. 4. Academic Press, Inc., San Diego, Calif.

18. Hooks, J. J., and C. J. Gibbs. 1975. The foamy viruses. Bacteriol. Rev. 39:169-185.

19. Hooks, J. J., C. J. Gibbs, E. C. Cutchins, N. G. Rogers, P. Lampert, and D. C. Gajdusek. 1972. Characterization and distribution of two new foamy viruses isolated from chimpanzees. Arch. Gesamte Virusforsch. 38:38-55.

20. Kaminsky, L. S., T. McHugh, D. Stites, P. Volperding, G. Henle, W. Henle, and J. A. Levy. 1985. High prevalence of antibodies to acquired immune deficiency syndrome (AIDS)-associated retrovirus (ARV) in AIDS and related conditions but not in other disease states. Proc. Natl. Acad. Sci. USA 82:5535-5539.

21. Keller, A., K. M. Partin, M. Löchelt, H. Bannert, R. M. Flügel, and B. R. Cullen. 1991. Characterization of the transcriptional trans activator of human foamy virus. J. Virol. 65:2589-2594.

22. Kupiec, J.-J., A. Kay, M. Hayat, R. Ravier, J. Peries, and F. Galibert. 1991. Sequence analysis of the simian foamy virus type 1 genome. Gene 101:185-194.

23. Kuritzkes, D. Personal communication.

24. Lanford, R. E., and J. S. Butel. 1984. Construction and characterization of an SV40 mutant defective in nuclear transport of $T$ antigen. Cell 37:801-813.

25. Lazinski, D., E. Grzadzielska, and A. Das. 1989. Sequence specific recognition of RNA hairpins by bactetiophage antiterminators requires a conserved arginine-rich motif. Cell 58:207-218.

26. Linial, M. L., and A. D. Miller. 1990. Retroviral RNA packaging: sequence requirements and implications. Curr. Top. Microbiol. Immunol. 157:125-152.

27. Löchelt, M., H. Zentgraf, and R. M. Flügel. 1991. Construction of an infectious DNA clone of the full-length human spumaretrovirus genome and mutagenesis of the bell gene. Virology 184:43-54.

28. Loh, P. C. 1993. Spumavirinae, p. 361-397. In J. A. Levy (ed.), The retroviridae, vol. 2. Plenum Press, New York.

29. Loh, P. C., B. C. Achong, and M. A. Epstein. 1977. Further biological properties of the human syncytial virus. Intervirology 8:204-217.

30. Mackett, M., G. L. Smith, and B. Moss. 1985. The construction and characterization of vaccinia virus recombinants expressing foreign genes, p. 191-211. In D. M. Glover (ed.), DNA cloning: a practical approach. IRL Press, Oxford.

31. Maurer, B., H. Bannert, G. Darai, and R. M. Flügel. 1988. Analysis of the primary structure of the long terminal repeat and the gag and pol genes of the human spumaretrovirus. J. Virol. 62:15901597.

32. Mergia, A., and P. A. Luciw. 1991. Replication and regulation of primate foamy viruses. Virology 184:475-482.

33. Muranyi, W., and R. M. Flūgel. 1991. Analysis of splicing patterns of human spumaretrovirus by polymerase chain reaction reveals complex RNA structures. J. Virol. 65:727-735.

34. Nassal, M. 1992. The arginine-rich domain of the hepatitis B virus core protein is required for pregenome encapsidation and productive viral positive-strand DNA synthesis but not for virus assembly. J. Virol. 66:4107-4116.

35. Nassal, M., and H. Schaller. 1993. Hepatitis B virus replication. Trends Microbiol. 1:221-228.

36. Netzer, K.-O., A. Rethwilm, B. Maurer, and V. ter Meulen. 1990. Identification of the major immunogenic structural proteins of human foamy virus. J. Gen. Virol. 71:1237-1241. 
37. Netzer, K.-O., A. W. Schliephake, B. Maurer, R. Watanabe, A. Aguzzi, and A. Rethwilm. 1993. Identification of pol-related gene products of human foamy virus. Virology 192:336-338

38. Neumann-Haefelin, D., U. Fleps, R. Renne, and M. Schweizer. 1993. Foamy viruses. Intervirology 35:196-207.

39. Neumann-Haefelin, D., A. Rethwilm, G. Bauer, F. Gudat, and H. zur Hausen. 1983. Characterization of a foamy virus isolated from Cercopithecus aethiops lymphoblastoid cells. Med. Microbiol. Immunol. 172:75-86.

40. Pan, L-Z. C. Cheng-Mayer, and J. A. Levy. 1987. Patterns of antibody response in individuals infected with the human immunodeficiency virus. J. Infect. Dis. 155:626-632.

41. Renne, R., E. Friedl, M. Schweizer, U. Fleps, T. Turek, and D. Neumann-Haefelin. 1992. Genomic organization and expression of simian foamy virus type 3 (SFV-3). Virology 186:597-608.

42. Rethwilm, A. Regulation of foamy virus gene expression. Curr. Top. Microbiol. Immunol., in press.

43. Rethwilm, A., G. Baunach, K.-O. Netzer, B. Maurer, B. Borisch, and V. ter Meulen. 1990. Infectious DNA of the human spumaretrovirus. Nucleic Acids Res. 18:733-738.

44. Rethwilm, A., G. Darai, A. Rösen, B. Maurer, and R. M. Flügel. 1987. Molecular cloning of the genome of the human spumaretrovirus. Gene 59:19-28.

45. Rikkonen, M., J. Peränen, and L. Kääriäinen. 1992. Nuclear and nucleolar targeting signals of semliki forest virus nonstructural protein nsP2. Virology 189:462-473.
46. Roberts, B. L., W. D. Richardson, and A. E. Smith. 1987. The effect of protein context on nuclear localization signal function. Cell 50:465-475.

47. Sambrook, J., E. F. Fritsch, and T. Maniatis. 1989. Molecular cloning: a laboratory manual, 2nd ed. Cold Spring Harbor Laboratory, Cold Spring Harbor, N.Y.

48. Sanger, F., S. Nicklen, and A. R. Coulson. 1977. DNA sequencing with chain-terminating inhibitors. Proc. Natl. Acad. Sci. USA 74:5463-5467.

49. Sharova, N., and A. Bukrinskaya. 1991. p17 and p17-containing gag precursors of input human immunodeficiency virus are transported into the nuclei of infected cells. AIDS Res. Hum. Retroviruses 7:303-306

50. Teich, N. 1984. Taxonomy of retroviruses, p. 25-208. In R. A Weiss, N. Teich, H. E. Varmus, and J. Coffin (ed.), RNA tumor viruses. Cold Spring Harbor Laboratory, Cold Spring Harbor, N.Y.

51. Temin, H. M. 1988. Mechanisms of cell killing/cytopathic effects by nonhuman retroviruses. Rev. Infect. Dis. 10:399-405.

52. Tuttleman, J. S., C. Pourcel, and J. Summers. 1986. Formation of the pool of covalently closed circular viral DNA in hepadnavirusinfected cells. Cell 47:451-460.

53. Varmus, H., and P. Brown. 1989. Retroviruses, p. 53-108. In D. E. Berg and M. M. Howe (ed.), Mobile DNA. American Society for Microbiology, Washington, D.C. 